\title{
Rezensionen online
}

\section{... von der Redaktion betreut (September - November 2018)}

Die Redaktion der Vierteljahrshefte für Zeitgeschichte arbeitet seit 2003 im Auftrag des Instituts für Zeitgeschichte München-Berlin mit dem Rezensionsjournal sehepunkte zusammen. Diese Kooperation findet nicht nur in den sehepunkten ihren Niederschlag, sondern auch in den Vierteljahrsheften selbst: In jedem Heft werden die von der Redaktion angeregten und betreuten Rezensionen angezeigt, die in den drei Monaten zuvor in den sehepunkten erschienen sind.

Dan Arav, Metim Lir'ot (Dying to See). War, Memory and Television in Israel 1967-1991, Tel Aviv 2017.

Rezensiert von: Tamar Amar-Dahl (Berlin) in sehepunkte 18 (2018), Nr. 9

www.sehepunkte.de/2018/09/32219.html

Matthias Bauer, Die transnationale Zusammenarbeit sozialistischer Parteien in der Zwischenkriegszeit. Eine Analyse der außenpolitischen Kooperations- und Vernetzungsprozesse am Beispiel von SPD, SFIO und Labour Party, Düsseldorf 2018.

Rezensiert von: Jakob Stürmann (Osteuropa-Institut, Freie Universität Berlin) in sehepunkte 18 (2018), Nr. 11

www.sehepunkte.de/2018/11/31740.html

Thomas M. Bohn / Aliaksandr Dalhouski / Markus Krzoska, Wisent-Wildnis und Welterbe. Geschichte des polnisch-weißrussischen Nationalparks von Białowieża, Köln / Weimar / Wien 2017.

Rezensiert von: Siarhei Novikau (Staatliche Linguistische Universität Minsk) in sehepunkte 18 (2018), Nr. 9

www.sehepunkte.de/2018/09/31601.html

Daniel Bohse, Die Entnazifizierung von Verwaltung und Justiz in Sachsen-Anhalt 1945 /46, Halle/Saale 2017.

Rezensiert von: Clemens Vollnhals (Hannah-Arendt-Institut für Totalitarismusforschung e.V. an der Technischen Universität, Dresden) in sehepunkte 18 (2018), Nr. 9

www.sehepunkte.de/2018/09/31117.html

Marcus Böick, Die Treuhand. Idee - Praxis - Erfahrung 1990-1994, Göttingen 2018.

Rezensiert von: Andreas Malycha (Institut für Zeitgeschichte München-Berlin) in sehepunkte 18 (2018), Nr. 10

www.sehepunkte.de/2018/10/31872.html 
Markus Börner / Anja Jungfer / Jakob Stürmann (Hgg.), Judentum und Arbeiterbewegung. Das Ringen um Emanzipation in der ersten Hälfte des 20. Jahrhunderts, Berlin / Boston 2018.

Rezensiert von: Angelika Timm (Berlin) in sehepunkte 18 (2018), Nr. 10

www.sehepunkte.de/2018/10/31842.html

Peter Brandt / Detlef Lehnert (Hgg.), Sozialdemokratische Regierungschefs in Deutschland und Österreich 1918-1983, Bonn 2017.

Rezensiert von: Felix Lieb (Institut für Zeitgeschichte München-Berlin) in sehepunkte 18 (2018), Nr. 9

www.sehepunkte.de/2018/09/31449.html

Rory Cormac, Disrupt and Deny. Spies, Special Forces, and the Secret Pursuit of British Foreign Policy, Oxford 2018.

Rezensiert von: Thomas Riegler (Wien) in sehepunkte 18 (2018), Nr. 10

www.sehepunkte.de/2018/10/31932.html

Ingo Cornils, Writing the Revolution. The Construction of „1968“ in Germany, Rochester / New York 2016.

Rezensiert von: Silja Behre (Minerva Institute for German History, Tel Aviv University) in sehepunkte 18 (2018), Nr. 9

www.sehepunkte.de/2018/09/31288.html

Andrew Demshuk, Demolition on Karl Marx Square. Cultural Barbarism and the People's State in 1968, Oxford / New York 2017.

Rezensiert von: Christian Rau (Institut für Zeitgeschichte München-Berlin) in sehepunkte 18 (2018), Nr. 9

www.sehepunkte.de/2018/09/31634.html

Andrew Dowling, The Rise of Catalan Independence. Spain's Territorial Crisis, London / New York 2018.

Rezensiert von: Reiner Tosstorff (Johannes Gutenberg-Universität, Mainz) in sehepunkte 18 (2018), Nr. 9

www.sehepunkte.de/2018/09/31213.html

Clemens Escher, „Deutschland, Deutschland, Du mein Alles!“ Die Deutschen auf der Suche nach ihrer Nationalhymne 1949-1952, Paderborn 2017.

Rezensiert von: Holger Löttel (Rhöndorf) in sehepunkte 18 (2018), Nr. 11

www.sehepunkte.de/2018/11/30506.html

Philipp Gassert, Bewegte Gesellschaft. Deutsche Protestgeschichte seit 1945, Stuttgart 2018.

Rezensiert von: Christoph Lorke (Westfälische Wilhelms-Universität, Münster) in sehepunkte 18 (2018), Nr. 11

www.sehepunkte.de/2018/11/32124.html 
Gedenkbuch für die Münchner Opfer der nationalsozialistischen „Euthanasie“Morde, hg. vom NS-Dokumentationszentrum München und dem Bezirk Oberbayern durch Michael von Cranach u.a., Göttingen 2018.

Rezensiert von: Moritz Fischer (Institut für Zeitgeschichte München-Berlin) in sehepunkte 18 (2018), Nr. 11

www.sehepunkte.de/2018/11/32069.html

Michael Gehler, Europa. Ideen, Institutionen, Vereinigung, Zusammenhalt, Reinbek 2017.

Rezensiert von: Bastian Knautz (Johannes Gutenberg-Universität, Mainz) in sehepunkte 18 (2018), Nr. 10

www.sehepunkte.de/2018/10/32403.html

Martin H. Geyer, Kapitalismus und politische Moral in der Zwischenkriegszeit. Oder: Wer war Julius Barmat?, Hamburg 2018.

Rezensiert von: Boris Barth (Karls-Universität, Prag) in sehepunkte 18 (2018), Nr. 11

www.sehepunkte.de/2018/11/31984.html

Maria Teresa Giusti, La Campagna di Russia 1941-1943, Bologna 2016.

Rezensiert von: Thomas Schlemmer (Institut für Zeitgeschichte München-Berlin) in sehepunkte 18 (2018), Nr. 11

www.sehepunkte.de/2018/11/29727.html

Martin Göllnitz, Der Student als Führer? Handlungsmöglichkeiten eines jungakademischen Funktionärskorps am Beispiel der Universität Kiel (1927-1945), Ostfildern 2018.

Rezensiert von: Gunnar Take (Institut für Zeitgeschichte München-Berlin) in sehepunkte 18 (2018), Nr. 10

www.sehepunkte.de/2018/10/32093.html

Thomas Großbölting, 1968 in Westfalen. Akteure, Formen und Nachwirkungen einer Protestbewegung, Münster 2018.

Rezensiert von: Ulf Teichmann (Ruhr-Universität Bochum) in sehepunkte 18 (2018), Nr. 10 www.sehepunkte.de/2018/10/31437.html

Todd H. Hall, Emotional Diplomacy. Official Emotion on the International Stage, Ithaca / London 2015.

Rezensiert von: Reinhild Kreis (Universität Mannheim) in sehepunkte 18 (2018), Nr. 9 www.sehepunkte.de/2018/09/29434.html

Andreas Jüngling, Alternative Außenpolitik. Der Freie Deutsche Gewerkschaftsbund der DDR und Franco-Spanien (1947 bis 1975), Berlin 2017.

Rezensiert von: Reiner Tosstorff (Johannes Gutenberg-Universität, Mainz) in sehepunkte 18 (2018), Nr. 10

www.sehepunkte.de/2018/10/31378.html 
Anke Kaprol-Gebhardt, Geben oder Nehmen. Zwei Jahrzehnte Rückübertragungsverfahren von Immobilien im Prozess der deutschen Wiedervereinigung am Beispiel der Region Berlin-Brandenburg, Berlin 2018.

Rezensiert von: Wolf-Rüdiger Knoll (Institut für Zeitgeschichte München-Berlin) in sehepunkte 18 (2018), Nr. 10

www.sehepunkte.de/2018/10/31941.html

Alex J. Kay, The Making of an SS Killer. Das Leben des Obersturmbannführers Alfred Filbert 1905-1990, Paderborn 2017.

Rezensiert von: Henning Pieper (Uelzen) in sehepunkte 18 (2018), Nr. 9

www.sehepunkte.de/2018/09/31635.html

Stephan Kieninger, The Diplomacy of Détente. Cooperative Security Policies from Helmut Schmidt to George Shultz, London / New York 2018.

Rezensiert von: Wilfried Loth (Münster) in sehepunkte 18 (2018), Nr. 11

www.sehepunkte.de/2018/11/31454.html

Carolin Kosuch, Missratene Söhne. Anarchismus und Sprachkritik im Fin de Siècle, Göttingen 2015.

Rezensiert von: Carsten Schapkow (Norman/Oklahoma) in sehepunkte 18 (2018), Nr. 10 www.sehepunkte.de/2018/10/31831.html

Franziska Meifort, Ralf Dahrendorf. Eine Biographie, München 2017.

Rezensiert von: Alexander Gallus (Technische Universität, Chemnitz) in sehepunkte 18 (2018), Nr. 9

www.sehepunkte.de/2018/09/30743.html

Stephen Milder, Greening Democracy. The Anti-Nuclear Movement and Political Environmentalism in West Germany and Beyond, 1968-1983, Cambridge 2017. Rezensiert von: Daniel Eggstein (Universität Konstanz) in sehepunkte 18 (2018), Nr. 10 www.sehepunkte.de/2018/10/30753.html

Raphael Minder, The Struggle for Catalonia. Rebel Politics in Spain, London 2017.

Rezensiert von: Reiner Tosstorff (Johannes Gutenberg-Universität, Mainz) in sehepunkte 18 (2018), Nr. 9

www.sehepunkte.de/2018/09/31213.html

Rolf-Dieter Müller, Reinhard Gehlen. Geheimdienstchef im Hintergrund der Bonner Republik. Die Biografie, Berlin 2017.

Rezensiert von: Armin Wagner (Dresden) in sehepunkte 18 (2018), Nr. 9

www.sehepunkte.de/2018/09/31212.html 
Helmut Müller-Enbergs / Thomas Wegener Friis (Hgg.), DDR-Spionage. Von Albanien bis Großbritannien, Frankfurt/M. 2018.

Rezensiert von: Andreas Hilger (Deutsches Historisches Institut, Moskau) in sehepunkte 18 (2018), Nr. 9

www.sehepunkte.de/2018/09/31764.html

Louis Pahlow / André Steiner, Die Carl-Zeiss-Stiftung in Wiedervereinigung und Globalisierung 1989-2004, Göttingen 2017.

Rezensiert von: Rainer Karlsch (Institut für Zeitgeschichte München-Berlin) in sehepunkte 18 (2018), Nr. 10

www.sehepunkte.de/2018/10/31265.html

Friedrich Pollock, Marxistische Schriften. Gesammelte Schriften Band 1. Herausgegeben von Philipp Lenhard, Freiburg/Brsg. / Wien 2018.

Rezensiert von: Gregor-Sönke Schneider (Hannover) in sehepunkte 18 (2018), Nr. 10

www.sehepunkte.de/2018/10/32173.html

Frank Reichherzer / Emmanuel Droit / Jan Hansen (Hgg.), Den Kalten Krieg vermessen. Über Reichweite und Alternativen einer binären Ordnungsvorstellung, Berlin / Boston 2018.

Rezensiert von: Liza Soutschek (Institut für Zeitgeschichte München-Berlin) in sehepunkte 18 (2018), Nr. 11

www.sehepunkte.de/2018/11/32175.html

Peter Romijn, Der lange Krieg der Niederlande. Besatzung, Gewalt und Neuorientierung in den vierziger Jahren, Göttingen 2017.

Rezensiert von: Rick Tazelaar (Institut für Zeitgeschichte München-Berlin) in sehepunkte 18 (2018), Nr. 11

www.sehepunkte.de/2018/11/31266.html

Jutta Rübke, Berufsverbote in Niedersachsen 1972-1990. Eine Dokumentation, Hannover 2018.

Rezensiert von: Jana Stoklasa (Leibniz Universität, Hannover) in sehepunkte 18 (2018), Nr. 9

www.sehepunkte.de/2018/09/32210.html

Mark Schiefer, Profiteur der Krise. Staatssicherheit und Planwirtschaft im Chemierevier der DDR 1971-1989, Göttingen 2018.

Rezensiert von: Rainer Karlsch (Institut für Zeitgeschichte München-Berlin) in sehepunkte 18 (2018), Nr. 11

www.sehepunkte.de/2018/11/32048.html 
Frank Schlöffel, Heinrich Loewe. Zionistische Netzwerke und Räume, Berlin 2018.

Rezensiert von: Stefan Vogt (Frankfurt/M.) in sehepunkte 18 (2018), Nr. 11

www.sehepunkte.de/2018/11/31851.html

Margrit Seckelmann / Johannes Platz (Hgg.), Remigration und Demokratie in der Bundesrepublik nach 1945. Ordnungsvorstellungen zu Staat und Verwaltung im transatlantischen Transfer, Bielefeld 2017.

Rezensiert von: Ewald Grothe (Gummersbach / Wuppertal) in sehepunkte 18 (2018), Nr. 9 www.sehepunkte.de/2018/09/30749.html

Tom Segev, David Ben Gurion. Ein Staat um jeden Preis, München 2018.

Rezensiert von: Bettina Sophie Weißgerber (Stuttgart) in sehepunkte 18 (2018), Nr. 11

www.sehepunkte.de/2018/11/31805.html

Edith Sheffer, Asperger's Children. The Origins of Autism in Nazi Vienna, New York / London 2018.

Rezensiert von: Rüdiger Graf (Zentrum für Zeithistorische Forschung, Potsdam) in sehepunkte 18 (2018), Nr. 10

www.sehepunkte.de/2018/10/32280.html

Werner Sonne, Leben mit der Bombe. Atomwaffen in Deutschland, Wiesbaden 2018.

Rezensiert von: Heiner Möllers (Zentrum für Militärgeschichte und Sozialwissenschaften der Bundeswehr, Potsdam) in sehepunkte 18 (2018), Nr. 10

www.sehepunkte.de/2018/10/31614.html

Winfried Süß / Malte Thießen (Hgg.), Städte im Nationalsozialismus. Urbane Räume und soziale Ordnungen, Göttingen 2017.

Rezensiert von: Eva Karl (Institut für Zeitgeschichte München-Berlin) in sehepunkte 18 (2018), Nr. 9

www.sehepunkte.de/2018/09/31719.html

Ray Takeyh / Steven Simon, The Pragmatic Superpower. Winning the Cold War in the Middle East, New York 2016.

Rezensiert von: Rolf Steininger (Innsbruck) in sehepunkte 18 (2018), Nr. 9

www.sehepunkte.de/2018/09/30563.html

Bastian Vergnon, Die sudetendeutschen Sozialdemokraten und die bayerische SPD 1945 bis 1978, Frankfurt/M. u. a. 2017.

Rezensiert von: Philipp Scheidle (München) in sehepunkte 18 (2018), Nr. 11

www.sehepunkte.de/2018/11/31593.html 
Nina Verheyen, Die Erfindung der Leistung, Berlin 2018.

Rezensiert von:Jan Kellershohn (Ruhr-Universität Bochum) in sehepunkte 18 (2018), Nr. 10

www.sehepunkte.de/2018/10/31670.html

Norman Weiß / Nikolas Dörr (Hgg.), Die Deutsche Gesellschaft für die Vereinten Nationen (DGVN). Geschichte, Organisation und politisches Wirken, 1952-2017, Baden-Baden 2017.

Rezensiert von: Steffen Fiebrig (Martin-Luther-Universität, Halle-Wittenberg) in sehepunkte 18 (2018), Nr. 10

www.sehepunkte.de/2018/10/31063.html

\section{Rezensionen zu Publikationen des IfZ (September - November 2018):}

Agnes Bresselau von Bressensdorf, Frieden durch Kommunikation. Das System Genscher und die Entspannungspolitik im Zweiten Kalten Krieg 1979-1982/83, Berlin / Boston 2015.

Rezensiert von: Gottfried Niedhart (Mannheim) in sehepunkte 18 (2018), Nr. 9 www.sehepunkte.de/2018/09/28208.html

Veronika Heyde, Frankreich im KSZE-Prozess. Diplomatie im Namen der europäischen Sicherheit 1969-1983, Berlin / Boston 2017.

Rezensiert von: Guido Thiemeyer (Düsseldorf) in sehepunkte 18 (2018), Nr. 11

www.sehepunkte.de/2018/11/30171.html 


\section{DE DE GRUYTER OLDENBOURG}

\section{JAHRBUCH FÜR WIRTSCHAFTSGESCHICHTE}

\section{BEIHEFTE}

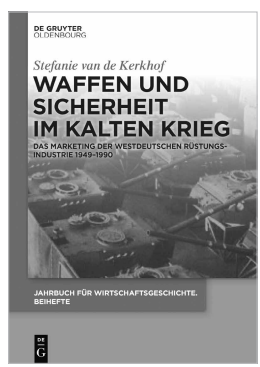

Stefanie van de Kerkhof WAFFEN UND SICHERHEIT IM KALTEN KRIEG

Das Marketing der westdeutschen Rüstungsindustrie 1949-1990

\section{Band 24}

09/2018. Ca. 448 S

Br. $€ 89,95$ [D]

ISBN 978-3-11-053907-3

eBook $€ 89,95$ [D]

PDF ISBN 978-3-11-054116-8

ePUB ISBN 978-3-11-053915-8

Die unternehmenshistorische Studie gewährt einen erstmalig tiefen und quellengesättigten Einblick in die Marketingstrategien der westdeutschen Waffenproduzenten im Kalten Krieg. Sie bietet eine methodisch breit angelegte und vergleichende Analyse im Sinne einer „Marketinggeschichte in der Erweiterung “. In innovativer Weise werden Sicherheitsforschung und Marketinggeschichte, Kultur- und Wirtschaftsgeschichte miteinander verbunden.

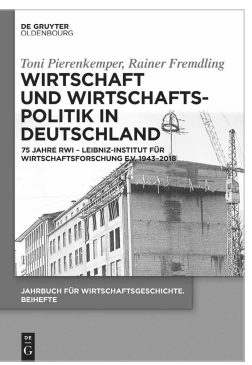

Rainer Fremdling, Toni Pierenkemper (Hrsg.)

WIRTSCHAFT UND WIRTSCHAFTSPOLITIK IN DEUTSCHLAND

75 Jahre RWI - Leibniz-Institut für

Wirtschaftsforschung e.V. 1943-2018

Band 22

07/2018. Ca. 512 S., $10 \mathrm{Abb}$

Br. $€ 99,95$ [D]

ISBN 978-3-11-056763-2

eBook Open Access

PDF ISBN 978-3-11-057055-7

ePUB ISBN 978-3-11-056773-1

Das Rheinisch-Westfälische Institut für Wirtschaftsforschung (RWI), 1943 mit der Verselbstständigung der „Abteilung Westen" des Deutschen Instituts für Wirtschaftsforschung (DIW) gegründet, wird umfassend von 1926 an über die Wiederbegründung und Umorientierung nach dem Krieg sowie die Neuausrichtung im neuen Jahrtausend bis 2018 dokumentiert und in die jeweiligen wirtschaftlichen, politischen und wissenschaftlichen Entwicklungen eingebettet.

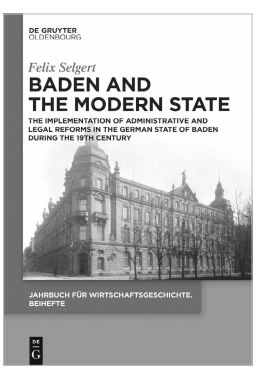

\author{
Felix Selgert \\ BADEN AND THE MODERN STATE \\ The Implementation of Administrative \\ and Legal Reforms in the German State \\ of Baden during the $19^{\text {th }}$ Century \\ Band 23 \\ 7/2018, ca. 208 S., 17 Abb. \\ Br. $€ 69,95[D]$ \\ ISBN 978-3-11-060079-7 \\ eBook $€ 69,95$ [D] \\ PDF ISBN 978-3-11-060265-4 \\ ePUB ISBN 978-3-11-059930-5
}

The bureaucracy's commitment to the public good and predictable decision making processes is an important prerequisite of economic growth. This study analyses how the rules and regulations that governed employment dismissal, promotion and remuneration of bureaucrats in the GrandDuchy of Baden during the first half of the $19^{\text {th }}$ century brought these features about. In doing so, the study builds on an extensive set of archival materials.

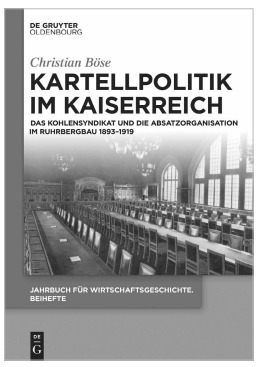

\author{
Christian Böse \\ KARTELLPOLITIK IM KAISERREICH \\ Das Kohlensyndikat und die \\ Absatzorganisation im Ruhrbergbau \\ 1893-1919 \\ Band 21 \\ 2018. XII, $335 \mathrm{~S}$ \\ Br. $€ 99,95[D]$ \\ ISBN 978-3-11-057431-9 \\ eBook € 99,95 [D] \\ PDF ISBN 978-3-11-057671-9 \\ ePUB ISBN 978-3-11-057438-8
}

Das Rheinisch-Westfälische Kohlen-Syndikat kontrollierte als zentrale Kartellorganisation des Ruhrbergbaus neben der Preis- und Produktionspolitik auch den Absatz der Zechen und baute dazu seit der Gründung 1893 einen mächtigen Vertriebsapparat auf. Ch. Böse stellt diesen Entwicklungsprozess dar, untersucht die Organisationsstrukturen und geht auf Konflikte ein, mit denen die Akteure bis zum Ende des Ersten Weltkrieges konfrontiert waren. 